\title{
Factors Influencing Tolerance of Ambiguity and Its Implications for Second Language Learning
}

\author{
Min Liu ${ }^{1, *}$ \\ ${ }^{1}$ College of Literature and Journalism, Sichuan University, Chengdu, Sichuan 610000, China \\ *Corresponding author. Email: lanyueliangpj@126.com
}

\begin{abstract}
In the process of second language learning, learners will inevitably encounter various situations of ambiguity, and the tolerance of ambiguity will directly affect the effectiveness of language learning. At present, tolerance of ambiguity has become an important index to evaluate foreign language learning and a key issue in the field of second language acquisition. In view of this, this paper examines the relevant variables that affect the tolerance of ambiguity, and then reveals that the tolerance of ambiguity is not a constant variable, but in a relatively dynamic development process. Finally, the paper points out that second language learners should adjust their tolerance of ambiguity appropriately and control the balance between quantity and quality of second language acquisition in order to achieve the best learning effect. At the same time, in the practice of second language teaching, teachers should also give full consideration to the balance of ambiguity tolerance, and effectively guide students to correctly treat and moderately tolerate ambiguity in learning.
\end{abstract}

Keywords: Tolerance of ambiguity, Second language, Learners.

\section{INTRODUCTION}

When learning a second language, learners will inevitably encounter all kinds of uncertain, strange and ambiguous language phenomena. What attitude should they take? Is it to avoid, reject and to be intolerable? Or to embrace and tolerate it? The different emotional reactions of learners reflect the problem of ambiguity tolerance or tolerance of ambiguity. Tolerance of ambiguity is one of the variables in the study of individual differences. "Not only is it one of the emotional factors, it can also reflect the learners' learning style. In the field of second language acquisition, tolerance of ambiguity refers to the learners' ability to rationally deal with ambiguity in learning stimulus. ${ }^{1}$

In fact, this concept was first put forward by American psychologist Frenkel Brunswik (1948). It means that when individuals or groups face a series of unfamiliar, complex, or inconsistent cues, the way they process ambiguities of environmental

1. Wei Wang, Niannian Zuo, Guonian Wang, et al. Introduction to applied linguistics [M]. Wuhan: China University of Geosciences Press, 2012:137 stimuli. ${ }^{2}$ Since then, foreign scholars have carried out a lot of research on this issue since the late 1950s. Among them, the research of Budner (1962) is of milestone significance. In his article "tolerance of ambiguity as a personal variable", he pointed out that ambiguity intolerance is a tendency to perceive ambiguous situations (that is, new, complex and difficult situations) as the source of threat. In recent years, foreign researchers have begun to redefine tolerance of ambiguity from the perspective of second language acquisition, and have conducted many discussions on its related variables and measurement methods. They found that tolerance of ambiguity was significantly correlated with foreign language learning anxiety, learning motivation and learning strategies, and had a significant impact on second language learning (Ehrman 1999; Scott et al. 2009). ${ }^{3}$ Compared with foreign countries, the research on tolerance of ambiguity by Chinese scholars is slightly weak. There is no systematic

2. Jie Shu. The influence of tolerance of ambiguity on foreign language learning $[\mathrm{J}]$. Journal of Southwest University for nationalities, 2005 (6).

3. Sumin Zhang. Multidimensional review and Reflection on the research of tolerance of ambiguity in the past 50 years $[\mathrm{J}]$. 
discussion on the works, and the number of journal papers is relatively limited. The current research results mainly include: Qingzong Zhang (2004) focused on the different situations of students with high and low tolerance of ambiguity using learning strategies; Sumin Zhang and Guiping Wang(2006) demonstrate the significant correlation between tolerance of ambiguity and English learning achievement; Sumin Zhang (2011) analyzes that tolerance of ambiguity has different effects on different learning tasks; Haichao Chang (2012) explores the mediating effect of learning strategies between tolerance of ambiguity and English proficiency through questionnaire survey, etc. Throughout the research in China and abroad, it is not difficult to find that most researchers are committed to defining the concept of tolerance of ambiguity or providing measurement methods. Unfortunately, "Tolerance of ambiguity, like other individual differences, is also affected by gender, society, culture, age and environment (Macaro 2008: 88-117)" ${ }^{4}$ By examining the relevant variables affecting the tolerance of ambiguity, this paper reveals that the tolerance of ambiguity is in a relatively dynamic development process. Second language learners should timely adjust their tolerance of ambiguity and control the balance between quantity and quality of second language acquisition in order to achieve the best learning effect.

\section{THE INFLUENCING FACTORS OF AMBIGUITY TOLERANCE}

Haichao Chang(2012) pointed out through empirical research that "tolerance of ambiguity is not only an emotion and personality (Arnold 1999), but also a learning style (Brown 2002), which is formed through long-term learning activities on the basis of individual physiology and under the influence of specific family, education and social culture." 5 Therefore, from the perspective of learners themselves, as one of the variables of individual differences, tolerance of ambiguity is also affected by other variables, such as personality type (introversion / extroversion), cognitive style (field independence / field dependence), family background, cultural background, etc. It is under the influence of these variables that learners show different cognitive affective tendencies in the face

4. Wei Wang, Niannian Zuo, Guonian Wang, et al. Introduction to applied linguistics [M]. Wuhan: China University of Geosciences Press, 2012: 137

5. Haichao Chang. The mediating effect of learning strategies on ambiguity tolerance and English proficiency [J]. of ambiguous situations. Some individuals have higher tolerance of ambiguity, while others have lower tolerance of ambiguity.

\subsection{Personality Types and Tolerance of Ambiguity}

Personality is one of the important factors that affect the learning effect, and it also affects the tolerance of learners to ambiguous situations. Generally speaking, learners' personality can be divided into introversion and extroversion. Extroverts "always seek stimulation, like to take risks, like to joke, and are very active"; on the contrary, introverts "always keep quiet" and "tend to avoid exciting things" (Eysenck, Chan, 1982:154). ${ }^{6}$ In the same second language learning environment, there are great differences in the performance of two kinds of learners when they face uncertain language phenomena.

Taking the English learning of Chinese learners as an example, when reading an English material, extroverted learners are generally able to tolerate these ambiguities if they encounter unfamiliar words or incomprehensible sentences, and focus their reading on the overall understanding of the passage, thus increasing the amount of language input. This kind of learners belong to people with high tolerance of ambiguity, However, introverted learners generally can't tolerate any ambiguity, and they have to look up the dictionary word by word to make it clear, which can easily affect the coherence of the whole discourse and the progress of understanding. This kind of students belongs to the typical people with low tolerance of ambiguity. Therefore, personality traits are closely related to tolerance of ambiguity, which in turn affects learners' language learning performance.

\subsection{Cognitive Style and Tolerance of Ambiguity}

Cognitive style is a term of cognitive psychology, which refers to "the habitual attitude and way that individuals prefer in the process of perception, memory, thinking and problem solving." 7 Among them, field independence and field dependence are one of human cognitive styles.

6. BingHuang. Introduction to second language acquisition [M]. Guangzhou: Guangdong Higher Education Press, 2004:178.

7. Lin Fan. Research on second language acquisition and foreign language teaching [M]. Qingdao: Ocean University of China Press, 2007:43 
In the field of second language acquisition research, these two cognitive styles are embodied in two different learning styles.

Witkin (1977) divides learning styles into two types: field independent and field dependent, and defines them as follows: "when presenting a dominant field, the individuals who are relatively field independent tend to overcome the organization of the field, or reconstruct it, while the individuals who are field dependent tend to adhere to the organization of the given field." ${ }^{8}$ In other words, in the presence independent model, individuals are less affected by the surrounding environment and are good at analyzing and constructing language materials; while in the presence dependent model, individuals are easily dominated by the external environment and are difficult to distinguish the specific part embedded in the presence, so they are better at learning language. It often depends on the pre organization of learning materials. Is it better to be field independent or field dependent? In fact, the two learning styles have their own advantages and disadvantages. A large number of studies have shown that field independent students are easy to achieve results in learning a second language in the classroom environment, while field dependent students are more dominant in the natural environment.

As for the relationship between the two learning styles and tolerance of ambiguity, there are also corresponding experimental arguments. For example, Chappelle (1986) once studied the relationship between field independence and tolerance of ambiguity, as well as the relationship between field independence and second language learning. He found that field independence subjects scored higher in English TOEFL. Through further research, he came to the conclusion that field independence is linguistic competence. It can predict the quality of foreign language learning. The reason why field independent individuals achieve better English performance is mainly due to their high tolerance of ambiguity. ${ }^{9}$ For example, when encountering ambiguous sentences, the learners with strong field independence usually have a higher tolerance of ambiguity, and are not easily disturbed by the surrounding variables, so

8. Lin Fan. Research on second language acquisition and foreign language teaching $[\mathrm{M}]$. Qingdao: Ocean University of China Press, 2007: 46.

9. Chupeng Wang. Applied Psycholinguistics: a study of foreign language learning psychology [M]. Changsha: Hunan Education Press, 1990: 125. they can distinguish ambiguity; while the field dependent learners have a relatively low tolerance of ambiguity, it is not so easy for them to distinguish the related items implied in ambiguity.

\subsection{Cultural Background and Tolerance of Ambiguity}

In previous studies, many scholars regard ambiguity tolerance as a personality variable, such as Frenkel Brunswik, Budner, Nedd, Marsh and so on. Since it is a personality variable, learners' tolerance of ambiguity is bound to be affected by the cultural factors of the country or region where the individual is located. At present, many studies have examined the relationship between tolerance of ambiguity and cultural background.

For example, Nedd and Marsh surveyed 218 male workshop managers in Auckland, New Zealand, who came from countries with different cultural backgrounds, and finally came to the conclusion that the order of ambiguity tolerance of these people from high to low was: European immigrants > European aborigines > Polynesian Asian immigrants > Polish aborigines. Hofstede uses the country Uncertainty Avoidance Index (UAI) to conduct a survey and finds that people from different cultural backgrounds have different tolerance for ambiguous situations. Durmysheva used 20 items of fuzzy tolerance scale, and developed eastern and Western questionnaires to investigate. Finally, it was found that oriental people's tolerance of ambiguity was relatively higher. ${ }^{10}$ From these studies, we can know that cultural background knowledge is closely related to ambiguity tolerance. Therefore, we have to consider the influence of cultural factors when we examine learners' tolerance of ambiguity. Some scholars point out that it is better to learn from McCrae's research paradigm of "cultural adaptation" in personality research to carry out cross-cultural research on tolerance of ambiguity, or this is also an interesting research direction.

\subsection{Learning Strategies and Tolerance of Ambiguity}

The so-called "learning strategy" refers to "the behavior or action that learners take in order to make language learning more successful, more autonomous and more enjoyable."(Oxford, 1989).

10. Baoguo Shi, Jiliang Shen, Jingjing Xu. Fuzzy tolerance: research review, current situation and prospect [J]. Psychology and behavior research, 2008 (6). 
According to Oxford (1990), learning strategies can be divided into direct strategies and indirect strategies. The former includes memory strategies, cognitive strategies and supplementary strategies, while the latter includes metacognitive strategies, affective strategies and social strategies. ${ }^{11} \mathrm{At}$ present, the existing researches mainly focus on the one-way influence of tolerance of ambiguity on the choice of learning strategies, that is, the level of tolerance of ambiguity determines how learners choose and use learning strategies. For example, McLain (1993) found that learners with high tolerance of ambiguity dare to take risks, often try to use new, dynamic and constantly changing language, and are also good at adopting new learning strategies. Qingzong Zhang (2004) confirmed that students with high tolerance of ambiguity can effectively use strategies, while students with low tolerance of ambiguity have great blindness and randomness in strategy selection. In fact, the relationship between tolerance of ambiguity and learning strategies is not one-way connection and influence, but complementary and interactive. The choice of learning strategies, in turn, will affect the adjustment of learners' tolerance of ambiguity. As usual, Haichao (2012) conducted a questionnaire survey on 502 non English major Chinese college students and found that metacognitive strategies and cognitive strategies have partial mediating effects between tolerance of ambiguity and English proficiency, and tolerance of ambiguity indirectly affects English proficiency through these two learning strategies. It also indirectly confirms the influence of learning strategies on ambiguity tolerance.

Here, we take cognitive strategy and metacognitive strategy as examples to demonstrate. Cognitive strategies are mainly used in the process of language learning, especially when completing specific classroom tasks. Their purpose is to contact, receive and transmit information, analyze and reason, and establish rules for input and output information (Oxford, 1990). Metacognitive strategies are mainly used to evaluate, manage and monitor the use of cognitive strategies. For example, sometimes we read an English article slowly in order to understand the content of the article. At this time, we mainly use cognitive strategies. But at other times, we can browse the articles quickly to understand the difficulty of the articles. Here we mainly use metacognitive

11. Cohen, A.D., Qiufang Wen. Strategies for learning and using a second language [M]. Beijing: foreign language teaching and Research Press, 2000: 15-16 strategies. Once learners have determined their learning strategies, they will subconsciously adjust their tolerance of ambiguity in the face of ambiguous language phenomena. In this regard, Haichao Chang (2012) also mentioned, "if learners have strong metacognitive awareness, metacognitive ability and self-monitoring ability in the face of ambiguous language phenomenon, we will not feel confused and puzzled. ${ }^{12}$ Second language learners use some learning strategies, the ultimate goal is to solve some problems encountered in language learning, or ambiguous situations, in order to be able to master the target language. In order to achieve this goal, learners must accordingly improve their tolerance of ambiguity. If learners fail to choose appropriate learning strategies effectively, they will easily become restless and intolerant of ambiguous language phenomena once they encounter ambiguous situations, which may lead to an instinctive rejection of the learning content and seriously affect the input and output of language materials. Therefore, learning strategies will in turn affect learners' tolerance of ambiguity.

To sum up, tolerance of ambiguity is a complex variable, which is not invariable. It has different degrees of correlation with learners' personality characteristics, cognitive style, learning strategies, cultural background, and learning motivation. The dynamic characteristics of some influencing factors determine that learners should adjust their tolerance of ambiguity accordingly. Of course, we cannot generally say that "high tolerance of ambiguity is good, and low tolerance of ambiguity is bad", which depends on the specific situation of ambiguity. As a second language learner, the most ideal state is to learn to adjust their tolerance of ambiguity by intervening in relevant variables in different ambiguity situations.

\section{TOLERANCE OF AMBIGUITY AND SECOND LANGUAGE LEARNING}

In the process of learning a second language, learners should be clearly aware of the differences between the target language and the mother tongue. When they are exposed to new and vague language stimuli, they should be allowed to have some certain uncertainty. Because the second language learning itself is "a process of constantly producing

12. Haichao Chang. The mediating effect of learning strategies on ambiguity tolerance and English proficiency [J]. Foreign languages, 2012 (2) 
high ambiguity", from the aspects of pronunciation, vocabulary, syntax, discourse, cultural background and so on, the differences between the target language and the mother tongue, the sense of distance and space-time will make the second language learners "often in a high degree of uncertainty". ${ }^{13}$

\subsection{Differences Between Target Language and Mother Tongue}

A study by Schachter (1974) shows that in foreign language practice, students tend to avoid structures that are quite different from their mother tongue, and excessively use structures similar to their mother tongue, that is, negative transfer of mother tongue occurs habitually. Schachter found that Chinese students did not like to use relative clauses. For example:

- There are so many Taiwan People * live around the lake.

- And there is a mountain * separates two lakes.

- There are so many tourists * visit there.

Why do Chinese students make the above syntactic mistakes? One of the reasons is that in the process of learning English, they find it difficult to learn relative clauses; that is, they encounter uncertain and unfamiliar language phenomena, so they choose to deliberately avoid the differences in some parameter values between English and Chinese. According to Schachter's analysis, Chinese is Left-branching, that is, the modifier is before the noun, while English is Right-branching, that is, the modifier is allowed after the noun. ${ }^{14}$

- The book Mary bought yesterday is really good.

- The book which Mary bought yesterday is very good.

Through the analysis of the above examples, it is found that learners should tolerate the differences between the target language and their mother tongue, actively adopt some strategies to reduce ambiguity situations, or learn to moderately enhance their own tolerance of ambiguity. At present, studies have shown that learners' tolerance

13. Qingzong Zhang. The influence of tolerance of ambiguity on the choice of foreign language learning strategies [J]. Foreign language teaching and research, 2004 (6)

14. Yu Wang. Second language acquisition and foreign language teaching $[\mathrm{M}]$. Tianjin: Tianjin University Press, 2014:70-71. of ambiguity is an important indicator to predict the effectiveness of target language learning. As Arnold and Brown (1999) pointed out, "successful language learning requires learners to at least partially accommodate the way of thinking and behavior of the target language." Ashuri and Fotovatnia (2010) also believe that "learners who accept the culture of the target language and have a positive attitude towards the target language and its application will be better at learning the target language." 15

\subsection{Implications of Tolerance of Ambiguity for Second Language Learning}

Second language acquisition researchers generally believe that high tolerance of ambiguity is not easy to be disturbed by ambiguous situations, and they are willing to take risks in second language learning, so they are easy to become successful language learners, while low tolerance of ambiguity cannot tolerate vague things, ambiguity makes them anxious, so they are prone to fossilization in target language learning. Reid hold this view. In fact, it is not so. Second language learners should maintain a clear, comprehensive and objective understanding of ambiguity tolerance. As defined by Ellis (1994), "when they are exposed to vague and brand-new language stimuli, they are neither depressed nor submissive to the ability of authority. It allows certain uncertainty and does not blindly pursue strict classification." 16

The main idea of Ellis is that learners should maintain a moderate tolerance of ambiguity. Too high or too low tolerance is not conducive to second language learning. For example, in oral English class, a person with low tolerance of ambiguity may be afraid that his pronunciation is not authentic or he is not sure which tense to use because he pays too much attention to the language form itself. Because he is afraid of making mistakes in expression, he will become timid and dare not speak out. For another example, in English intensive reading class, a person with high tolerance of ambiguity is prone to "excessive tolerance of ambiguity, no serious thinking and Research on any problem, ambiguous words, will

15. Sumin Zhang. Multidimensional review and Reflection on the research of tolerance of ambiguity in the past 50 years [J]. Foreign languages, 2012 (2).

16. Yan Yin. The influence of "tolerance of ambiguity" on English learning and Its Enlightenment on Classroom English Teaching $[\mathrm{J}]$. Foreign language circles, 2012 (2). 
lead to no understanding." ${ }^{17}$ Generally speaking, excessive intolerance of ambiguity will prematurely reject those contradictory phenomena, which is harmful to foreign language learning; excessive tolerance of ambiguity will lead to swallowing dates and hinder the absorption of meaningful content (Brown, 2002).

Because of this, second language learners need to correctly grasp a "degree", and know how to properly adjust their tolerance of ambiguity according to different learning contents, learning tasks and different ambiguity situations. For example, when the tolerance of ambiguity is low, learners can consciously use some compensation techniques, such as "free discussion, retelling stories, role play, interpretation, finding synonyms, information gap practice, skimming and inferring the meaning of words according to context"; when the tolerance of ambiguity is too high, learners can also "take some similar precise reading and translation to regulate." 18 There are many adjustment measures in this respect, depending on the specific ambiguity situation. If a learner can be aware of his tolerance of ambiguity and cultivate a reasonable tolerance of ambiguity, he will give full play to his initiative and creativity, deal with the obstacles in language learning flexibly, and complete the construction of the second language successfully, thus becoming a successful language learner.

\section{CONCLUSION}

As the cognitive, psychological and emotional factors of learners become the key issues in the field of second language acquisition, the degree of ambiguity tolerance has attracted more and more attention from scholars.Researchers in China and abroad have analyzed the behavioral characteristics, cognitive tendency and emotional attitude of people with different degrees of tolerance of ambiguity from the aspects of cognition, emotion and learning style. In the process of the development of the theory of tolerance of ambiguity, some measurement tools with ideal reliability and validity have been produced. Although there are many measurement tools to divide the tolerance of ambiguity into high and low degrees, in fact, the

17. LifeiWang. Cognitive style research and its implications for foreign language teaching [J]. Shandong foreign language teaching, 1998 (4).

18. Jie Shu. The influence of tolerance of ambiguity on foreign language learning $[\mathrm{J}]$. Journal of Southwest University for nationalities, 2005 (6). tolerance of ambiguity is not a constant variable. Due to the constraints of individual differences and other variables, it may present a dynamic process in the process of second language learning.

At the same time, too high or too low tolerance of ambiguity is not a good phenomenon, which is not conducive to second language learning, which is also very enlightening to the practice of second language teaching. In other words, teachers must fully consider the balance of students' tolerance of ambiguity. On the one hand, teachers should teach students in accordance with their aptitude according to their individual differences, "guide them to correctly treat and moderately tolerate the new language and cultural phenomena in learning, improve their tolerance of ambiguity, use appropriate learning strategies and learning methods, experience foreign language learning happily, and improve learning effectiveness." ${ }^{19} \mathrm{On}$ the other hand, teachers should also grasp the problem of "degree" to avoid overcorrection. Too high or too low tolerance of ambiguity is not conducive to second language learning. The ideal state is moderate tolerance of ambiguity, but it is difficult to grasp this ideal state, which needs teachers and students to explore slowly in the longterm running in.

\section{AUTHORS' CONTRIBUTIONS} Liu.

This paper is independently completed by Min

\section{REFERENCES}

[1] Lin Fan. Research on second language acquisition and foreign language teaching [M]. Qingdao: China Ocean University Press, 2007

[2] Bing Huang. Introduction to second language acquisition [M]. Guangzhou: Guangdong Higher Education Press, 2004

[3] Cohen, A.D., Qiufang Wen. Strategies for learning and using a second language [M]. Beijing: foreign language teaching and Research Press, 2000

[4] Chupeng Wang. Applied Psycholinguistics: a study of foreign language learning psychology [M]. Changsha: Hunan Education Press, 1990

19. Qingzong Zhang. The influence of tolerance of ambiguity on the choice of foreign language learning strategies [J] Foreign language teaching and research, 2004 (6) 
[5] Wei Wang, Niannian Zuo, GuonianWang, et al. Introduction to applied linguistics [M]. Wuhan: China University of Geosciences Press, 2012

[6] Yu Wang. Second language acquisition and foreign language teaching $[\mathrm{M}]$. Tianjin: Tianjin University Press, 2014

[7] HaichaoChang. The mediating effect of learning strategies on ambiguity tolerance and English proficiency $[\mathrm{J}]$. Foreign languages, 2012 (2)

[8] Baoguo Shi, Jiliang Shen, Jingjing Xu. Fuzzy tolerance: research review, current situation and prospect $[\mathrm{J}]$. Psychology and behavior research, 2008 (6)

[9] Jie Shu. The influence of tolerance of ambiguity on foreign language learning $[\mathrm{J}]$. Journal of Southwest University for nationalities, 2005 (6)

[10] Lifei Wang. Cognitive style research and its implications for foreign language teaching $[\mathrm{J}]$. Shandong foreign language teaching, 1998 (4)

[11] Ju Xu. Universal grammar and second language acquisition $[\mathrm{J}]$. Foreign language teaching, 2006 (1)

[12] Yan Yin. The influence of "tolerance of ambiguity" on English learning and Its Enlightenment on Classroom English Teaching [J]. Foreign language circles, 2012 (2)

[13] Qingzong Zhang. The influence of tolerance of ambiguity on the choice of foreign language learning strategies $[\mathrm{J}]$. Foreign language teaching and research, 2004 (6)

[14] Sumin Zhang. Multidimensional review and Reflection on the research of tolerance of ambiguity in the past 50 years $[\mathrm{J}]$. Foreign language circles, 2012 (2) 